\title{
Une minute pour convaincre. L'expression politique à l'épreuve des débats télévisés des primaires de 2016-2017
}

One minute to convince. Political expression and the test of televised debates of the 2016-2017 French primaries

Un minuto para convencer. Expresión política a prueba de los debates televisados de las primarias 2016-2017

\section{Éric Treille}

\section{OpenEdition}

Journals

Édition électronique

URL : https://journals.openedition.org/mots/23276

DOI : $10.4000 /$ mots. 23276

ISSN : 1960-6001

Éditeur

ENS Éditions

Édition imprimée

Date de publication : 5 juillet 2018

Pagination : $35-53$

ISSN : 0243-6450

Référence électronique

Éric Treille, « Une minute pour convaincre. L'expression politique à l'épreuve des débats télévisés des primaires de 2016-2017 », Mots. Les langages du politique [En ligne], 117 | 2018, mis en ligne le 05 juillet 2020, consulté le 22 avril 2022. URL : http://journals.openedition.org/mots/23276 ; DOI : https:// doi.org/10.4000/mots.23276 


\section{Une minute pour convaincre. L'expression politique à l'épreuve des débats télévisés des primaires de 2016-2017}

En rassemblant 8,5 millions de téléspectateurs, le dernier débat de la primaire de la droite et du centre a été un succès. Il a même su dépasser, en termes d'audimat, l'échange inauguré par le Parti socialiste (PS) en 2011, confirmant ainsi l'intérêt des Français pour la politique et leur curiosité pour cette nouvelle procédure de désignation.

Pour réitérer ce captage de l'attention du public, le parti Les Républicains (LR) a érigé le précédent socialiste en modèle, en se calant sur la forme et le nombre de débats qui avaient été privilégiés en 2011, consacrant alors ce qui est devenu en soi un «nouveau genre télévisuel» (Richard, Sandré, 2013) avec ses règles d'exposition et sa dramaturgie propre. En choisissant en plein accord avec les directions des chaînes des formats volontairement corsetés à la seconde près, LR et le PS ont également souhaité se prémunir d'un double danger : produire, comme Europe-Écologie-Les-Verts (EELV), des échanges aseptisés à force d'autocontrôle, ou dévoiler au grand jour des divisions internes, comme ce fut le cas en 2006 lors de la première primaire socialiste. Contrairement au système américain où les débats sont nombreux, rugueux et surtout étalés sur une longue période, le système des primaires à la française a été organisé sur un temps court et selon des modalités pratiques volontairement contraignantes afin de ne pas écorner par avance l'image du candidat désigné (Lefebvre, Treille, 2016).

La tenue de débats sérieux au risque de l'ennui a dès lors été le prix à payer pour prévenir les «faux pas» (Neveu, 1998) et éviter que n'éclatent les rivalités et les polarités idéologiques trop tranchées. Pour répondre au cadre d'expression conjointement arbitré - une minute par intervention, quinze à dix-sept minutes par débattant - et au nombre élevé de compétiteurs en présence - sept pour la droite comme pour la gauche -, les candidats ont souhaité dans leur majorité « interdire tout propos désobligeant » ${ }^{1}$ en favorisant l'usage

1. Le Parisien, 27 janvier 2017, interview de Benoît Hamon sur l'organisation du débat télévisé de l'entre-deux-tours de la primaire de la Belle Alliance populaire.

Arènes UMR 6051 (Univ Rennes - EHESP - CNRS)

eric.treille@laposte.net

Mots. Les langages du politique $\mathrm{n}^{\circ} 117$ juillet $2018 \bullet 35$ 
de phrases courtes à visée pédagogique, ou petites phrases phatiques, des "discours sans adversaires » (Juhem, 2001) ramassés, permettant de condenser leur programme par l'emploi de marqueurs spécifiques, laissant majoritairement aux «outsiders» le recours aux petites phrases polémiques et aux logiques éristiques (Gauthier, 2013).

Nous souhaitons montrer que cette fragmentation contrainte de l'expression politique a résumé l'ambiguïté de cette configuration partisane et médiatique «hybride» (Chadwick, 2017), qui devait à la fois départager et rassembler. Dans ce cadre, l'énonciation de petites phrases doit moins être lue comme un symptôme de la « dégradation de la parole publique » (Krieg-Planque, OllivierYaniv, 2011) que comme une adaptation de la mécanique de la synthèse activée par les formations politiques pour discipliner le pluralisme intra-partisan (Sawicki, 2017) à ce nouveau format d'émission argumentative (Treille, 2013).

On s'efforcera de mettre en valeur les stratégies rhétoriques de réduction déployées par les candidats des primaires de la droite et de la Belle Alliance populaire pour «affadir» leur discours (Brasart, 1994), de la fabrication des argumentaires en amont à l'édulcoration télévisée de l'éloquence tribunitienne, du surlignage électronique des petites phrases jusqu'à l'acmé des débats de l'entre-deux-tours, permettant à cette nouvelle technologie de désignation de demeurer, selon le néologisme d'Arnaud Montebourg, un scrutin de «coopétition», contraction de coopération et de compétition².

\section{Les stratégies mimétiques de LR et du PS ou la confirmation d'un «nouveau genre télévisuel »}

Longtemps considéré comme contraire à la culture politique française, le système des primaires a été érigé en modèle de sélection des candidats à l'élection présidentielle par EELV, LR et le PS. En s'imposant d'abord en 2006 sous une forme fermée, puis plus sûrement dans un cadre ouvert en 2011, cette nouvelle procédure de désignation n'a pas seulement transformé les modes de régulation de la concurrence interne à la gauche (Lefebvre, 2011), elle a également profondément modifié la fabrique médiatique d'un exercice jusqu'alors confiné aux seuls cercles dirigeants ou militants (Lefebvre, Treille, 2016), soulignant en cela l'importance des débats des primaires dans la visibilité de la campagne présidentielle3.

2. Cette recherche s'appuie sur une étude comparative des stratégies rhétoriques développées par les candidats lors des huit débats télévisés des primaires de la droite et de la Belle Alliance populaire, ainsi que sur leur réception dans la presse écrite (pour la primaire de la droite et du centre : le 12 janvier, débat organisé parTF1, RTL et Le Figaro ; le 3 novembre, BFM TV et I-Télé; le 17 novembre, France 2 et Europe 1 ; le 24 novembre, TF1 et France 2. Pour la primaire de la Belle Alliance populaire : le 12 janvier, débat organisé par TF1, LCI, Public Sénat, RTL et L'Obs; le 15 janvier, BFM TV, RMC, I-Télé et RMC; le 19 janvier, France 2 et Europe 1 ; le 25 janvier, TF1, France 2 et France Inter).

3. Le succès des débats des primaires a conduit les chaînes de télévision, et au premier chef TF1, 
Depuis la première consultation organisée par le PS en 2006, le centre de gravité de la présélection du chef de l'État s'est ainsi déplacé de la scène strictement partisane aux plateaux télévisés (Leroux, Riutort, 2013). Alors que les partis politiques peinaient à s'exposer, allant parfois jusqu'à rechercher «l'audimat désespérément », selon l'expression de la journaliste de BFM-TV Apolline de Malherbe4, le débat qui avait vu s'affronter Martine Aubry et François Hollande le 15 septembre 2011 sur France 2 avait très largement battu TF1 et son émission MasterChef, renouvelant de manière inédite le rôle traditionnellement joué par les émissions argumentatives dans les mobilisations électorales (Trognon, Larrue, 1994 ; Leroux, Riutort, 2011).

Cette médiatisation nouvelle de ce qui relevait traditionnellement des coulisses du politique, et pour 2006 des seules chaînes d'information en continu et de LCP, n'a cependant été concédée par les partis qu'au prix de la maîtrise de l'organisation des débats. En effet, l'externalisation du cadre délibératif de la désignation des candidats ne s'est pas traduite par un abandon des conditions de fabrication de ces confrontations télévisées. Mieux : ces débats ont dans les faits constitué un exercice médiatique et politique co-construit entre les chaînes de télévision et les organisateurs des primaires ("Les plateaux télévisés, l'autre front de la guerre des droites», Le Monde, 10 février 2016). Du choix des thèmes abordés à la couleur du décor, de la taille des pupitres à la mise en scène, tout a été négocié jusqu'au moindre détail, au risque, comme l'avait déjà souligné Jean-Pierre Elkabbach lors de la primaire socialiste de 2006, de revenir à «la télévision de Brejnev».

De fait, compte tenu des enjeux strictement internes du scrutin et du nombre élevé des inscrits dans la compétition - sept concurrents à droite comme à gauche en lieu et place d'un duel -, les organisateurs des primaires ont mis en place un dispositif d'encadrement du temps de parole extrêmement resserré (une minute à une minute vingt pour se présenter et pour conclure, trente secondes pour échanger, quarante à quarante-cinq secondes pour répondre - un «droit de suivre» décompté du temps de parole - et quinze à dix-sept minutes pour l'ensemble de l'émission), quitte à transformer ces débats en successions «d'interviews croisées » et de « discours monologaux» (Sandré, 2017).

En bousculant ainsi l'ordonnancement du «script » des classiques confrontations d'entre-deux-tours de l'élection présidentielle (Kerbrat-Orecchioni, 2017), le genre « débats télévisés des primaires» n'a pas reproduit à l'identique «l'événement discursif» que constituent les «débats pour l’Élysée» (Barbet, Mayaffre, 2009). À l'image des cahiers des charges rédigés lors des primaires

à innover en organisant pour la première fois dans l'histoire de la Ve République un débat entre les principaux candidats à l'élection présidentielle en amont de la traditionnelle diffusion du débat d'entre-deux-tours, soulignant ainsi un effet inédit de la «primarisation » de la vie politique sur le fonctionnement des médias.

4. A. Malherbe, Politiques cherchent audimat, désespérément, Paris, Albin Michel, 2007. 
américaines, l'imposition de ces exigences a souligné la spécificité d'un scrutin destiné au grand public qui ne devait toutefois pas constituer une menace pour les partis et les candidats en lice.

\section{Un devoir de concision ou la parole politique fragmentée}

Cette contrainte du temps a eu deux conséquences. Elle a d'abord imposé aux journalistes le rôle ingrat de «contrôle de la parole» (Oger, 2003). Comme l'a souligné Élizabeth Martichoux, animatrice du premier débat de la primaire de la droite, «nous n'avions qu'une minute par candidat. La contrainte du temps ne nous autorisait guère à les relancer, même si certaines réponses m'ont poussée à le faire »5. Elle a ensuite mis en demeure les candidats de respecter scrupuleusement ce très strict cadrage de l'expression, «l'objectif est d'être clair et concis " 6 selon l'équipe du maire de Bordeaux, un voyant lumineux ayant même été disposé afin de les alerter sur les dépassements du chronomètre.

Si cette contrainte a restreint les capacités d'action des animateurs des débats, elle a cependant constitué un atout paradoxal pour les candidats en limitant fortement les moments d'interruption ou les risques de se voir couper la parole (Sandré, 2009). Elle les a ainsi obligés à organiser en amont une fausse spontanéité en isolant des «fragments de discours» (Krieg-Planque, 2013) pouvant à la fois s’insérer dans des cadres programmatiques déjà prédéfinis et constituer des traits suffisamment distinctifs par rapport aux autres compétiteurs.

De fait, l'exercice des primaires a conduit les candidats à bâtir des stratégies rhétoriques de réduction de phrases déjà formellement «petites ». Contrairement aux paroles prononcées dans le cadre de meetings, pour lesquels l'anticipation des «détachements énonciatifs» (Maingueneau, 2011) est une routine de travail, ce mode extrêmement fragmenté d'intervention a transformé l'ensemble des discours en condensés d'expressions politiques.

Cette narration morcelée n'est pas en soi spécifique. L'invention de ces débats partisans s'est intégrée dans un mouvement plus général « d'abréviation des unités de contenu » (Krieg-Planque, 2011) que l'on retrouve déjà dans la presse écrite gratuite ou encore dans les médias électroniques. Elle diffère cependant sur un point : les candidats de la primaire, pourtant habitués à s'exprimer dans des formats courts, ont dû apprendre à «surasserter » des formulations qui avaient déjà, en raison de leur brièveté, l'apparence formelle de petites phrases, obligeant ainsi selon ce proche d'un candidat à «bosser des formules $» 7$.

5. Le Monde, 8 et 9 janvier 2017.

6. 20 Minutes, 13 octobre 2016.

7. Le Parisien, 20 septembre 2016. 


\section{L’amont des débats télévisés ou la fabrication collective d'argumentaires}

La phase préparatoire des débats a été essentielle pour aider les candidats à condenser leurs propos. Pour cela, des propositions de formules qui «disent ce qu'il faut dire » (Krieg-Planque, 2013) ont été fabriquées par les différentes équipes de campagne pour guider les paroles dans la confrontation - les moments ouverts - comme dans la récitation - les moments plus fermés -, servir de «béquilles argumentatives» dans les échanges interdiscursifs et encadrer les énonciations non dialogiques du débat, notamment lors des introductions et des conclusions.

À rebours des pratiques habituelles, ces phases préparatoires ne sont cependant pas restées dans les coulisses de l'émission ${ }^{8}$, mais ont également servi de supports de communication, certains candidats n'hésitant pas à médiatiser le récit de ces entraînements oratoires. À droite, l'équipe de Bruno Le Maire a organisé en privé un débat fictif avec de faux pupitres et une lampe symbolisant le voyant lumineux utilisé par TF1. Des proches y jouaient le rôle des compétiteurs, et le président du conseil départemental de l'Eure, Sébastien Lecornu, n'a pas hésité à relater dans la presse ses impressions de double de Nicolas Sarkozy. À gauche, Manuel Valls s'est exercé «en situation », tout en soulignant que «ce n'est pas du bachotage "9. Jean-Luc Bennahmias a préparé de son côté les débats avec un «ami metteur en scène» :

Ce n'est pas de l'impro. Il faut convaincre des millions de téléspectateurs avec des prises de paroles très courtes. [...] J'ai travaillé quelques trucs car je parle comme une mitraillette. ${ }^{10}$

Arnaud Montebourg a travaillé ses interventions avec des coachs pour adapter son «body language» au format spécifique des débats. Comme l'a souligné son directeur de campagne, «sur la forme, on y est. Le plus difficile est de savoir comment on synthétise le contenu qu'on veut faire passer ${ }^{11}$. Benoît Hamon s'est astreint au même exercice : "Je ne sais pas faire court, c'est un vrai problème $»^{12}$, concède-t-il. Comme le soulignait son directeur de campagne, Mathieu Hanotin, «il y a un travail de synthèse à faire pour rendre nos propositions intelligibles sur une minute trente $»^{13}$.

En revanche, les candidats n'ont pas souhaité exposer ces documents préparatoires au moment des débats. Le masquage de leurs fiches a même fait l'objet de négociations avec les chaînes de télévision : des pupitres opaques

\footnotetext{
8. "Jeux de rôles, fiches, footing et autres recettes », Le Figaro, 13 octobre 2016.

9. Le Parisien, 12 janvier 2017.

10. Libération, 23 janvier 2017.

11. Le Parisien, 12 janvier 2017.

12. Le Monde, 27 janvier 2017.

13. Le Parisien, 12 janvier 2017.
} 
ont ainsi été installés et les caméramans ont eu l'interdiction de filmer les notes des différents compétiteurs.

La mise au jour de la fabrique politique est donc encore restée limitée. Un candidat peut se montrer entouré dans la phase préparatoire des débats, mais il peut en revanche refuser de dévoiler l'ensemble des procédés qui ont participé à la production de son discours. La magie «auctoriale» (Maingueneau, 2011) de la prise de parole devait être conservée et l'effet « candidature en nom collectif» gommé (Le Bart, 2012), obligeant chacun des candidats à reléguer à l'arrière-plan les éléments techniques qui pouvaient rendre inauthentiques les arguments partagés, tout comme les «écrivants » (Ollivier-Yaniv, 2003) qui avaient contribué à leur rédaction.

\section{La communication numérique des petites phrases ou le surlignage électronique du discours politique}

Le petit écran n'a pas été le seul espace de diffusion des confrontations des primaires. Pour renforcer l'efficacité performative des arguments utilisés, les équipes web des candidats ont également été destinataires de ces formules pré-rédigées. Comme le souligne Dorine, membre de l'équipe de campagne numérique de Manuel Valls, «aujourd'hui, on ne peut pas se passer de Facebook et de Twitter. Il faut y être et appuyer ce que le candidat va dire ${ }^{14}$. Des petites phrases déjà sélectionnées ont été diffusées en temps réel, dédoublant typographiquement la formulation orale des déclarations jugées les plus fortes. Pour certaines propositions phares du programme, des visuels avaient même été préparés pour être diffusés sur Tumblr (Avecmanuelvalls.trumblr.com par exemple), renforçant le surlignage souhaité.

Le caractère récitatif des débats, notamment dans les phases introductives et conclusives, est pour beaucoup dans l'usage démultiplicateur de ces «mobilisations de clavier» (Badouard, 2013). Les argumentaires ont servi de dérouleurs à la fois pour les candidats et pour les équipes de campagne qui pouvaient y puiser des formules quasiment sans modification ou nécessité de validation politique (Blanchard, 2009). Ce mode d’organisation, intégrant dans un même mouvement les supports télévisés et numériques, a aussi permis aux candidats de mieux contrôler les reprises souhaitées en organisant, sans le travail d'intercesseur des journalistes, la propre fragmentation de leur discours.

Comme pour les séances d'entrainement des candidats, le travail des équipes web a également été mis en scène et intégré en tant que tel dans les dispositifs de campagne. En cela, les candidats n'ont fait que reproduire des méthodes de communication déjà utilisées en 2012 lors de la dernière élec-

14. 20 Minutes, 26 janvier 2017. 
tion présidentielle. L'équipe de $M$. Valls a ainsi organisé une « riposte party »15 (Theviot, 2016) dans un pub irlandais du $11^{\mathrm{e}}$ arrondissement de Paris. Ce choix de rendre visible l'activisme en ligne du candidat a permis de multiplier les scènes d'exposition médiatique et de donner aux journalistes des solutions pratiques de mise en image de procédés techniques (Treille, 2013). Il a également permis à ces équipes web de bénéficier des réactions in situ de la salle - rires, applaudissements... - pour sélectionner les petites phrases les plus remarquables, soulignant l'hybridation du « genre de papier» (Krieg-Planque, 2011) avec les nouvelles techniques de communication numérique et la « porosité » qui existe entre des formes de communication variées (Poupard, 2005). Il a enfin permis d'anticiper le travail « d'arbitrage journalistique » qu'effectue la presse $^{16}$ et d'éviter ainsi que ne soient désignés des vainqueurs par anticipation (Leroux, Riutort, 2011), montrant en cela la forte intégration par les équipes de campagne des règles d'écriture de l'information politique selon les codes du «journalisme de course de chevaux».

\section{Le détachement du revenu universel d'existence ou une proposition programmatique à effets polémiques}

La rédaction d'argumentaires en amont a également eu pour fonction d'installer des thèmes de campagne. Profitant du temps d'exposition qui lui fut attribué, B. Hamon a choisi de détailler longuement sa mesure fétiche, le revenu universel d'existence, détachant volontairement ce fragment programmatique de son projet présidentiel au travers de phrases courtes à visée pédagogique, ou petites phrases phatiques ${ }^{17}$.

En installant d'emblée ce thème, B. Hamon a créé une ligne de clivage «à effets» (Brasart, 1994) à la fois distinctive pour l'électorat des primaires - en valorisant la «dimension épidictique» de son discours - et répulsive pour les autres candidats. Comme le souligna l'un de ses proches, le député de Paris Pascal Cherki, "plus on nous attaque, plus on se renforce ${ }^{18}$. Lors du premier débat télévisé du 12 janvier 2017, Vincent Peillon l’interrogea sur le coût du dispositif : «Le revenu universel, c'est 400 milliards d'euros. Qui les paiera?»

15. Expression forgée en 2012 par Romain Pigenel, responsable du pôle Influence au QG de François Hollande, afin de médiatiser les dispositifs de «live-tweets » mis en œuvre par l'équipe internet du candidat socialiste à l’élection présidentielle.

16. Le journal Le Parisien a mis en place un système de notation de 1 à 10 des candidats des primaires de la droite et de la gauche selon 5 critères - la précision, le style, le fair-play, la combativité et la meilleure formule.

17. "J'ai une conviction : nous vivons des bouleversements inédits. La manière dont nous consommons, le travail se transforment. [...] Il faut, pour cela, tourner la page avec les vieilles recettes. Je veux proposer un chemin où il y aura un nouveau pilier pour notre protection sociale : le revenu universel d'existence ».

18. L'Opinion, 12 janvier 2017. 
A. Montebourg lui reprocha « de baisser les bras sur la lutte contre le chômage », une petite phrase déjà utilisée lors d'interviews ${ }^{19}$. M. Valls souligna qu'il voulait «une société de travail», une formulation plus lisse que celle utilisée en réunions publiques visant «la société de l'assistanat et du farniente».

Le thème du revenu universel s'est imposé dans les quatre débats télévisés de la primaire de la Belle Alliance populaire, quitte à surligner le procédé de la reprise comme méthode de composition privilégiée des débatteurs, parfois dans le même échange, et comme technique de neutralisation de ce qui pouvait s'apparenter à des formes d'éloquence trop visibles ${ }^{20}$. Lors de la troisième confrontation du 19 janvier 2017, l'ancien Premier ministre a ainsi usé deux fois d'un parallélisme de construction déjà utilisé, se risquant alors à s'auto-citer : «je ne veux pas d'une gauche qui fait des propositions à crédit pour perdre à l'arrivée totalement son crédit » et «je ne veux pas d'une gauche qui fait des promesses illimitées pour demain se confondre avec les impôts illimités». A. Montebourg cibla à la suite de V. Peillon le coût du dispositif. En réponse, $B$. Hamon répliqua qu' « on peut négocier avec son banquier, pas avec notre planète », variante de la petite phrase qu'il avait déjà utilisée une fois avec succès lors du premier débat du 15 janvier 2017 : «les marchés qui détiennent notre dette, nous pouvons négocier avec eux. La planète non ».

Afin de favoriser les reports de voix et compenser l'absence de ralliements expressément exprimés, cette stratégie du discours rapporté a également visé des propos tenus par des candidats non retenus pour le second tour. Pour dénoncer le financement du revenu universel, $M$. Valls reprit ainsi mot pour mot le slogan de campagne d'A. Montebourg : «Je suis le candidat de la feuille de paie et je ne veux pas que mon ami Benoît soit le candidat de la feuille d'impôt». En y accolant un second segment caractérisant par harmonie imitative le programme de son concurrent, l'ancien Premier ministre a pu alors donner des gages verbaux et des signaux d'allégeance indirects à ce nouvel électorat à conquérir en reformulant ses éléments de «reprise diaphonique»(Vion, 2006)21.

\section{Pousser à la faute ou la petite phrase, arme langagière privilégiée des candidats outsiders}

Pour imposer sa stratégie de campagne, l’ordre de passage a constitué un atout certain pour le premier désigné. Jean-François Copé a ainsi pleinement profité

19. A. Montebourg déclara à propos du projet de revenu universel qu'il s'agissait d'un «cataplasme social dans le champ stratosphérique de l'irréalisme", Le Monde, 12 janvier 2017.

20. V. Peillon a emprunté le procédé déjà utilisé par F. Hollande lors de son débat contre N. Sarkozy, une anaphore construite autour de la phrase "un président de gauche, c'est... ».

21. Cette opération de ventriloquie politique fut en revanche plus assumée à droite. Interrogé sur l'enjeu de l'identité lors du dernier débat d'entre-deux-tours le 24 novembre, F. Fillon s'est ainsi mis dans les pas de N. Sarkozy et a déclaré : "Quand on vient dans la maison d'un autre, par courtoisie, on ne prend pas le pouvoir, on respecte cet autre. » 
d'un tirage au sort favorable pour cibler dès le départ son principal adversaire, N. Sarkozy, sans permettre à ce dernier de répondre, utilisant alors le véhicule des primaires pour solder son départ contraint de la tête de l'UMP et l'enquête sur l'affaire Bygmalion : «ll y a dix ans en 2007, j'avais espéré en la rupture que proposait Nicolas Sarkozy pour notre pays». Malgré les efforts de l'ancien chef de l'État pour souligner ce qui le rapprochait de ses concurrents - «nous avons gouverné ensemble pendant cinq ans \} { } ^ { 2 2 } \text { -, le maire de Meaux l'a obligé à adop- } ter une stratégie défensive et à user de son précieux temps de parole pour contrer un candidat crédité de $1 \%$ des intentions de vote dans les sondages ${ }^{23}$.

Cette stratégie de déstabilisation fut révélatrice du mode de gestion du temps imposé à chacun des candidats. Comme le confiait ce proche de B. Le Maire, «dans ce débat, on est acteur pendant 15 minutes, spectateur pendant 75 minutes ${ }^{24}$. De fait, l'énonciation d'une petite phrase a non seulement pour objectif d'engager un échange, mais également, en cas notamment d'absence de réponse, de trahir les émotions internes de son contradicteur, qui faute de temps ou parce qu'il refuse de se faire interpeller doit apprendre à travailler ses silences (Sandré, 2011) et son langage corporel (Coulomb-Gully, 2009). En cela, la crispation de N. Sarkozy, filmée avec force détails pendant l'intervention inaugurale de J.-F. Copé, a constitué un moment fort du premier débat. En masquant difficilement son exaspération, l'ancien président de la République renforçait ainsi la portée du message de son concurrent et esquissait en amont la nature de l'usage qu'il allait faire de son devoir de réponse.

L'attaque n'a cependant pas réussi à renforcer la candidature de J.-F. Copé ni, plus largement, à bousculer l'ordre des prétendants établi par les sondages. De fait, l'énonciation d'une petite phrase ne peut être détachée du positionnement hiérarchique de son locuteur. Malgré l'égalité proclamée, la majorité des compétiteurs n'a fait que de la figuration médiatique et électorale. Pis, la présence de certains de ces "petits candidats » n'a pas eu d'autres fonctions que de permettre d'afficher une «diversité factice » 25 à la fois politique - la place des partenaires au sein de coalitions - et sexuée - l'inscription très difficile de Nathalie Kosciusko-Morizet et la présence sans nécessité de parrainages de Sylvia Pinel comme symptômes d'un « rappel à l'ordre genré » (Matonti, 2017) d'une scène partisane restée quasi exclusivement masculine ${ }^{26}$.

Comme le souligne Alice Krieg-Planque, «l'existence de petites phrases est également suscitée par des déterminants médiatiques» (Krieg-Planque, 2011, p. 29). La référence implicite à l'affaire Bygmalion et à ses prolongements

22. Le Figaro, 14 octobre 2016.

23. "La loi anti-burqa, pardon Jean-François, ce n'est pas toi qui nous l'as imposée et tu étais bien incapable d'imposer ni au Premier ministre ni au président de la République quoi que ce soit, pas plus hier qu'aujourd'hui. »

24. Libération, 12 octobre 2016.

25. "Le quart d'heure de célébrité des outsiders », Le Monde, 18 janvier 2017.

26. "À gauche, le pouvoir reste une affaire d'hommes», Le Monde, 20 janvier 2017. 
partisans fut ainsi rapidement noyée dans le débat par d'autres affaires de plus grande portée électorale. B. Le Maire fut le premier, à travers sa proposition de rendre public son casier judiciaire, à engager un réquisitoire contre ses concurrents et en premier lieu contre A. Juppé, même si «ça ne vise personne mais ça concerne tout le monde ». Le maire de Bordeaux dut alors riposter : "Tout le monde connaît la condamnation qui m'a été infligée. Aujourd'hui, les Français ont la décision au bout de leur bulletin de vote. S'ils estiment que ma faute me disqualifie, ils ne m'éliront pas». N. Sarkozy, doublement visé, a répondu à cette proposition - tout en ciblant également F. Fillon, auteur de la petite phrase «Qui imagine le général de Gaulle mis en examen?» - en rétorquant que ce "ne sont pas des déclarations qui honorent ceux qui les prononcent », puis en rappelant qu'il n'avait jamais été condamné : "Cinq non-lieux, des heures de télévision, des heures de bassesses, de calomnies. Rien». J.-F. Copé a repris le même procédé litotique de «discours sans adversaires» (Juhem, 2001) que B. Le Maire en déclarant ne «viser personne» tout en affirmant : "Oui, c'est vrai, si j'avais été mis en examen, je n'aurais pas été candidat». Dernier candidat à s'exprimer, F. Fillon put alors clore cette séquence à la fois politique et judiciaire en promettant d'être le président «du courage, de la vérité, de l'action et l'honnêteté », une "vertu » auto-attribuée (Kerbrat-Orecchioni, 2017)27 qu'il reprendra également lors du second débat - «choisissez la rigueur, choisissez l'honnêteté».

En choisissant tous les deux de faire précéder leur intervention de formules d'atténuation (Oger, Ollivier-Yaniv, 2006), B. Le Maire et J.-F. Copé évitaient à la fois de cibler nominativement leurs concurrents et cherchaient à pousser A. Juppé et N. Sarkozy à la faute. En dépit de ces attaques, les candidats visés n'ont cependant pas dévié de leur position. Comme l'a souligné Benoist Apparu, le porte-parole du maire de Bordeaux, «Alain Juppé doit dérouler ses propres thèmes. La posture du chicaya, il doit la laisser aux autres pour apparaître comme celui qui prend de la hauteur ${ }^{28}$. Compte tenu du temps de parole distribué à chacun, les candidats favoris ne pouvaient se permettre de perdre la «bataille pour le contrôle de l'agenda (Gerstlé, 2017) de la campagne. Comme le résumait ce proche de N. Kosciusko-Morizet, «rien ne peut bouger dans un débat aussi millimétré ${ }^{29}$. Dans ce cadre, il s'agissait moins de faire preuve d'éloquence que de ne pas commettre d'impairs en récitant des argumentaires comprimés en quelques phrases. Un lieutenant d’A. Juppé pouvait même affirmer «match nul, match gagné ${ }^{30}$. La distinction est surtout restée le privilège

27. Cette stratégie, gagnante au moment des débats télévisés, s'est cependant transformée en piège politique pour F. Fillon lorsque Le Canard enchaîné publia son enquête sur le statut professionnel de son épouse.

28. Le Monde, 13 octobre 2016.

29. Libération, 16 octobre 2016.

30. Le Journal du dimanche, 16 octobre 2016. 
des «petits candidats » comme Jean-Frédéric Poisson ou J.-L. Bennahmias, au risque de susciter la réprobation des « grands candidats ", troublés dans leurs exposés par des interventions jugées par trop intempestives.

\section{Des débats à sept ou les impossibles combats singuliers de N. Sarkozy et de M. Valls}

L'organisation inédite de débats à sept a également eu pour conséquence de fragmenter l'espace des échanges en plusieurs sous-confrontations d'inégales valeurs politiques en lieu et place des classiques duels de second tour.

Ainsi, alors que N. Sarkozy s'était préparé à engager le fer contre le maire de Bordeaux, B. Le Maire, N. Kosciusko-Morizet et J.-F. Copé ont choisi lors du second débat du 3 novembre 2016 de concentrer leurs attaques sur l'ancien président de la République, l'obligeant alors à répondre pour défendre son propre bilan présidentiel. B. Le Maire démina sa stratégie de stigmatisation de François Bayrou en moquant la «dream team» qui gouvernait en 1993. De même, il interpella l'ancien président sur la composition de son gouvernement («Nicolas, l'ouverture a beaucoup troublé notre électorat»), l'obligeant à sortir de sa réserve et à utiliser son temps de parole contre un «petit candidat » : "Tu étais dans ce gouvernement et tu as même postulé pour être Premier ministre». N. Kosciusko-Morizet dénonça également sa campagne identitaire : «On a eu le burkini, on a eu les Gaulois, et maintenant on nous fait le coup de l'invasion centriste. Pas de compromission avec les thèses du FN. On ne fricote pas avec leurs idées, on ne braconne pas sur leurs terres. Il y a un risque que le prochain mandat présidentiel soit empoisonné par l'extrême droite. Je vous invite à un vote antidote ». F. Fillon relia ses propres thématiques de campagne au sujet Bayrou : "Ill y a six millions de chômeurs, 100 \% de dettes et, le sujet majeur, c'est le sort du maire de Pau? Moi, je ne rentre pas dans ce pugilat». Dans ce cadre désormais déminé par les renforts des autres candidats et les lapsus sur «François Bayroin », illustrés par de nombreux plans de coupe sur un François Baroin amusé, A. Juppé eut alors toute latitude pour moquer « cette fixation » et dénoncer ce qu'il appela « une querelle subalterne».

Compte tenu de ces stratégies d'escarmouche, N. Sarkozy n'a pas pu engager la confrontation qu'il souhaitait avec A. Juppé, le duel désiré se transformant dans les faits en offensive anti-sarkoziste. Pis, contraint de répondre, il laissa à la fois les « petits candidats » utiliser son statut d'ancien chef de l'État comme faire-valoir pour asseoir leur crédibilité présidentielle, A. Juppé confirmer son propre rôle de rassembleur et F. Fillon émerger, au point d'installer un match à trois en lieu et place du duel initialement promis.

À gauche, la principale figure d'unité du premier débat fut paradoxalement une figure absente, F. Hollande jouant le rôle dévolu à N. Sarkozy. B. Hamon 
évoqua son "sentiment d'inachevé», A. Montebourg jugea le quinquennat «difficile à défendre», V. Peillon attaqua quant à lui vivement le livreconfession Un président ne devrait pas dire ça... Les secrets d'un quinquennat31, en déclarant «je ne crois pas que ce soit le bavardage [...] qui donne la dimension d'un homme d'État». Lors du second débat, M. Valls prit plus classiquement la place occupée par N. Sarkozy. Comptable du bilan du quinquennat, l'ex-Premier ministre fut mis en minorité par les anciens responsables du Nouveau Parti Socialiste, à nouveau unis pour la circonstance. Au sein de ce trio, V. Peillon s'est tout particulièrement distingué, obligeant M. Valls à utiliser une partie de son temps de parole pour se justifier et endosser contre son gré les habits du sortant en lieu et place du statut de primo-candidat. Face à cette stratégie, la répétition à sept reprises de «je veux» dans sa conclusion n'a pas suffi à contrebalancer les petites phrases très écrites de l'eurodéputé : la freudienne Schiefertafel « la vie, ce n'est pas une ardoise magique », le parallélisme de construction «critiquer tes discours, ce n'est pas critiquer la France» ou encore la litote «j'ai le sentiment que les Français étaient peut-être plus généreux que leurs dirigeants».

\section{Le refus du pugilat ou des débats de « coopétition »}

À la différence du système nord-américain, où les échanges entre les candidats rythment avec régularité la campagne et permettent de «tester» chacun des postulants, le système français des primaires a délibérément placé les débats à la fin de la compétition, et ce dans un calendrier très resserré. Le PS a ainsi programmé ses trois débats en l'espace d'une semaine seulement, quelques jours avant l'organisation de la consultation. Dans ce cadre, les débats télévisés ont constitué une acmé paradoxale du scrutin. Attendue par la presse comme une "foire d'empoigne ${ }^{32}$, la confrontation télévisée a surtout eu pour but d'éviter les déchirements et de permettre le rassemblement le plus large possible du parti autour du candidat désigné, transformant ainsi les primaires en exercice de «coopétition », pour reprendre le néologisme d’A. Montebourg évoqué plus haut (Lefebvre, Treille, 2016).

À l'image des candidats de la primaire socialiste de 2011, les participants aux débats des deux primaires ont tenu à respecter ce cahier des charges, au risque d'apparaître «scolaires »33. Comme l'a précisé A. Juppé lors d’un déplacement à Tours, «faire campagne, ce n'est pas lâcher une incongruité tous les

31. G. Davet et F. Lhomme, Un président ne devrait pas dire ça... Les secrets d'un quinquennat, Paris, Stock, 2016.

32. "Sept candidats, deux droites», Le Monde, 15 octobre 2016.

33. Ibid. 
jours pour faire parler de soi ! $\$ 34$. Comme le rappelait également ce proche du maire de Bordeaux, «l'invective est pénalisante pour celui qui la profère » 35 . Un proche d'A. Montebourg adopta la même réserve : "Le premier qui tape sur un autre est disqualifié. Il faut être percutant, vif, offensif dans la proposition mais ne pas donner de leçons». Pour un autre collaborateur de candidat, «tout le monde a intérêt à être canalisé »36. L'adoption de ce ton policé a cependant eu un prix : les échanges ont banni les petites phrases trop définitives qui pouvaient être reprises, une fois le vote terminé, par les adversaires du représentant choisi. En définitive, les principaux candidats à refuser cette stratégie furent les « outsiders». Comme le souligna N. Kosciusko-Morizet : «À quoi ça sert de débattre si on ne se lâche pas un peu? On n'allait pas rester figés quand même!»

\section{Les journalistes pris pour cible ou la critique du régime médiatique}

Ce refus de se démarquer par la petite phrase de trop a cependant souffert une importante exception. Dans le prolongement de la position qu'avait déjà adoptée F. Fillon sur le plateau de France 2 vis-à-vis de Charline Vanhoenacker37, les journalistes ont été pris pour cible à plusieurs reprises par les différents compétiteurs, de droite et plus modérément de gauche, au risque de rompre le "contrat de communication » pourtant préalablement établi avec les chaînes de télévision (Charaudeau, 2005).

Le dernier débat de la primaire de la droite et du centre s'est tout particulièrement distingué en raison de l'intervention de N. Sarkozy à l'encontre de David Pujadas, lorsque ce dernier, relayant les affirmations de Ziad Takieddine, interrogea l'ancien président sur un possible financement libyen de sa campagne électorale de 2007 : «Quelle indignité! Vous n’avez pas honte de donner écho à un homme qui fait de la prison. Ce n'est pas l'idée, voyez-vous, que je me fais du service public ». N. Kosciusko-Morizet fit de même en répondant à un journaliste «peut-être qu'on peut éviter de parler pendant vingt minutes d'Emmanuel Macron » ou encore B. Le Maire lorsqu'il répondit à une question de J.-P. Elkabbach : «Vous connaissez déjà le résultat de dimanche? Vous savez ce que vont voter les Français? Ce sont les Français qui jugeront dimanche, pas vous! Je n'ai pas de leçons à recevoir de votre part sur ma candidature».

34. Le Monde, 20 et 21 novembre 2016.

35. 20 Minutes, 13 octobre 2016.

36. Le Figaro, 12 janvier 2017.

37. Interpellé par la journaliste de France Inter à la fin de L'Émission politique (France 2, 27 octobre 2016), F. Fillon déclara : «J'ai beaucoup de respect pour Charline, je la trouve très drôle. Je ne suis pas totalement convaincu que ce soit particulièrement approprié de conclure une émission politique de cette manière.» 
À la fin de l'émission, F. Fillon refusa d'interpeller l'un de ses contradicteurs comme l'y invitait D. Pujadas, respectant en cela le canevas préalablement coconstruit entre les journalistes et les équipes de campagne : «Vous nous coupez la parole sur des sujets absolument fondamentaux pour que l'on s'interpelle les uns les autres. C'est tout le problème de ces débats, une conception en termes de spectacle et pas en termes de fond $»$.

Si les débats opposant les compétiteurs de gauche furent plus pauvres en dénonciations du rôle de la presse, certaines attaques ont cependant pu émerger des échanges. B. Hamon répliqua ainsi sèchement au journaliste qui l'interrogeait au sujet de l'Europe : «Si Manuel Valls veut être candidat en Allemagne et moi en Angleterre, on vous fera signe ». V. Peillon a également accusé $M$. Valls d'être le représentant des «forces politiques et des forces de presse». A. Montebourg fut cependant celui qui se distingua le plus lorsqu'il visa la journaliste Laurence Ferrari en parlant de «votre chaîne, le propriétaire de votre chaîne » pour dénoncer la concentration des médias et le rôle joué par Vincent Bolloré au sein d'l-Télé.

La construction de règles du jeu communes ne garantit donc en rien l'établissement d'un climat d'apaisement. Malgré des modalités d'exposition et des sujets co-négociés, les candidats de la primaire ont critiqué les journalistes qui les interrogeaient, usant ainsi d'une «rhétorique (désormais) obligée de la communication politique ${ }^{38}$ qui doit cependant moins à un « dysfonctionnement interactionnel » (Sandré, 2011) localisé et circonstanciel - même si cette dénonciation tactique de tiers a permis de suspendre momentanément la confrontation - qu'à une transformation plus profonde des relations entre le monde politique et le monde journalistique.

\section{Le petit écran contre l'estrade ou l'édulcoration télévisée de l'éloquence tribunitienne}

Les débats d'entre-deux-tours ont confirmé le souhait initialement émis de privilégier l'aridité des développements au détriment de l'acidité des échanges. Compte tenu des écarts importants de voix entre les candidats arrivés en tête - F. Fillon a obtenu 44,1\% des suffrages, seize points devant A. Juppé -, les confrontations n'avaient de fait plus la même portée. Le maire de Bordeaux pouvait alors déclarer «j'ai toujours eu pour toi de l'amitié et de l'estime» et le député de Paris répondre «ce débat ne doit pas être celui de la division ». En écho à gauche, $M$. Valls pouvait également affirmer «je veux dire mon plaisir d'être ici avec B. Hamon pour qui j'ai de l'amitié et du respect».

38. Sur la dénonciation de la presse pendant la campagne présidentielle de 2017, voir notamment l'interview d'Arnaud Mercier : «Les politiques libèrent la parole des esprits agressifs et violents », Libération, 15 juillet 2017. 
Dans ce climat «fair-play»39, le ton n'est qu'exceptionnellement monté, moins cependant pour déstabiliser un adversaire que pour édulcorer des arguments utilisés les jours précédents. Le débat de l'entre-deux-tours de la primaire de la droite et du centre fut ainsi l'occasion de reproduire de manière "affadie» (Brasart, 1994) des propos tenus en tribunes, au risque parfois de l'abscons lorsque les sujets motivant le recours à ces petites phrases n'étaient pas explicitement rappelés. Ce fut notamment le cas lorsque le débat aborda la question de l'avortement qui avait suscité la veille de vifs affrontements. F. Fillon déclara: «Le procès qui m’a été fait ces derniers jours n'est pas correct [...]. Ce débat est absurde, nous n'aurions pas dû l'avoir». A. Juppé, au lieu de revenir sur ses propos - F. Fillon incarnerait une vision « extrêmement traditionaliste, pour ne pas dire rétrograde» de la société - se contenta alors de dire «je n'ai fait aucun procès», préférant revenir sur les «boules puantes» dont il avait été l'objet sans pourtant en préciser la nature - notamment la campagne menée sur les réseaux sociaux dans laquelle le maire de Bordeaux était surnommé «Ali Juppé» (Mercier, 2017). Il reprit ainsi, mot pour mot, une formule déjà utilisée en meeting à Toulouse deux jours plus tôt : «J'aurais aimé qu'un certain nombre de mes compétiteurs condamne ces attaques ignominieuses».

Le débat d'entre-deux-tours de la primaire de la Belle Alliance populaire a reproduit le même type de passes d'armes. Attaqué par M. Valls sur l'antenne de France Info à propos d'une phrase prononcée sur l'absence de mixité dans certains cafés de banlieue, et plus durement encore par l'un de ses proches, Malek Boutih, député de l'Essonne («Benoît Hamon est en résonance avec une frange islamo-gauchiste », 20 Minutes, le 24 janvier 2017), B. Hamon riposta en dénonçant les «arguments du Front national » sans jamais cibler directement son adversaire socialiste («j’ai bien vu le procès qui m’a été fait. Florian Philippot, Marine Le Pen »), alors que la veille sur France 2 il regrettait que «des personnes de [sa] famille politique relaient des attaques de l'extrême droite».

En changeant de scène d'exposition, le discours politique change de format d'énonciation. Même si les meetings d'aujourd'hui sont fortement marqués par la médiatisation de la vie publique et l'arrivée des chaînes d'information en continu, les petites phrases prononcées devant des militants, même filmées, n'ont pas nécessairement pour finalité d'être reprises in extenso devant un public plus large, la théâtralisation de «fort petites paroles» (Brasart, 1994) et le recours aux mots à effets que commande l'éloquence tribunitienne pouvant apparaître décalés sur un plateau de télévision (Cossart, 2010) ou pis, résolument contre-productifs lorsque l'enjeu principal des primaires n'est plus de départager deux finalistes mais de préparer le rassemblement post-scrutin derrière le candidat désigné. 
C'est donc tout le paradoxe des débats télévisés des primaires. Conçus pour départager à ciel ouvert des candidats jusqu'alors désignés dans l'entresoi partisan, ces échanges ne pouvaient pas pour autant virer au pugilat et alimenter ainsi le stéréotypage de l'affrontement cathodique du second tour de l'élection présidentielle (Barbet, Mayaffre, 2009). Dans ce cadre, la fabrique de petites phrases majoritairement consensuelles ou visant des tiers non politiques comme les journalistes a joué un rôle clé pour résoudre la difficile équation de ce dispositif «hybride», mi-partisan mi-médiatique (Chadwick, 2017). Par construction politique et en raison du formatage télévisuel co-adopté, les débats des primaires ne devaient pas refléter l'entièreté des dispositifs langagiers engagés pendant la campagne, notamment les plus négatifs d'entre eux (Labbé, Monière, 2014). Ils ont au mieux constitué une caisse de résonance atténuée de propos d'estrade ou de discours monologaux prononcés sur d'autres antennes, obligeant les candidats - tout particulièrement les favoris - non seulement à ramasser leurs propositions programmatiques dans des abrégés pré-rédigés, mais également à lisser leurs réparties afin de neutraliser les éventuels risques de polémiques (Oger, Ollivier-Yaniv, 2006) et permettre in fine la nécessaire unité de l'après-second tour (Lefebvre, Treille, 2016).

\section{Références}

BADOUARD Romain, 2013, "Les mobilisations de clavier. Le lien hypertexte comme resSources des actions collectives en ligne », Réseaux, n¹81, p. 87-117.

Barbet Denis, Mayaffre Damon, 2009, «2007. Débats pour l'Élysée», Mots. Les langages du politique, n०89, p.5-9.

BLANCHARD Gersende, 2009, "La mise en œuvre de la communication électronique des partis politiques français : le poids des pratiques politiques et organisationnelles», Les enjeux de l'information et de la communication, volume 2009, p. 3-15.

BRASART Patrick, 1994, "Petites phrases et grands discours. (Sur quelques problèmes de l'écoute du genre délibératif sous la Révolution française) ", Mots. Les langages du politique, $\mathrm{n}^{\circ}$ 40, p. 106-112.

CHAdwick Andrew, 2017, The Hybrid Media System. Politics and Power, Oxford, Oxford University Press.

Charaudeau Patrick, 2005, Le discours politique : les masques du pouvoir, Paris, Vuibert.

COSSART Paula, 2010, Le meeting politique. De la délibération à la manifestation (18681939), Rennes, Presses universitaires de Rennes.

CoulomB-Gully Marlène, 2009, « Le corps présidentiel. Représentation politique et incarnation dans la campagne présidentielle française de 2007 ", Mots. Les langages du politique, $\mathrm{n}^{0} 89$, p. 25-38.

GAUthIER Gilles, 2013, "La manœuvre éristique dans le débat d'entre-deux-tours de l'élection présidentielle française de 2012 », dans Présidentielle 2012. Une communication politique bien singulière, P.J. Maarek éd., Paris, L'Harmattan.

GERSTLÉ Jacques, 2016, «Débat des primaires : la bataille pour le contrôle de l'agenda », Theconversation.com, https://theconversation.com/debat-des-primaires-la-bataillepour-le-controle-de-lagenda-67086 (consulté le 20 janvier 2018) 
JUHEM Philippe, 2001, «La légitimation de la cause humanitaire : un discours sans adversaires", Mots. Les langages du politique, nº65, p. 9-27.

Kerbrat-Orecchion I Catherine, 2017, Les débats de l'entre-deux-tours des élections présidentielles françaises. Constantes et évolutions d'un genre, Paris, L'Harmattan.

KRIEG-PlANQUE Alice, 2013, «Un discours sur prescription : les “argumentaires" des partis politiques comme éléments de cadrage de la parole», Argumentation \& analyse du discours, $n^{0} 10$, http://journals.openedition.org/aad/1438 (consulté le 20 janvier 2018).

- 2011, «Les petites phrases : un objet pour l'analyse des discours politiques et médiatiques », Communication \& langages, $\mathrm{n}^{0} 168$, p. 23-41.

Krieg-Planque Alice, Ollivier-Yaniv Caroline, 2011, «Poser les “petites phrases” comme objet d'étude», Communication \& langages, nº 168, p.17-22.

LABBÉ Dominique, MonıÈre Denis, 2014, « Ne votez pas pour l'autre! La spirale de la négativité», dans 2012. La campagne présidentielle. Observer les médias, les électeurs, les candidats, J. Gerstlé, R. Magni Berton éd., Paris, L'Harmattan.

LE BART Christian, 2012, La politique en librairie. Les stratégies de publication des professionnels de la politique, Paris, Armand Colin.

LefEBVRE Rémi, 2011, Les primaires socialistes. La fin du parti militant, Paris, Raisons d'agir.

LefebVRe Rémi, Treille Éric, 2016, Les primaires ouvertes en France. Adoption, codification, mobilisation, Rennes, Presses universitaires de Rennes.

LEROuX Pierre, RIUTORT Philippe, 2013, La politique sur un plateau. Ce que la télévision fait à la représentation, Paris, PUF.

- 2011, "Les émissions de divertissement : de nouveaux lieux de valorisation des petites phrases? », Communication \& langages, n0168, p. 69-80.

MAINGUENEAU Dominique, 2011, "Les petites phrases : sur une petite phrase "de" Nicolas Sarkozy. Aphorisation et auctorialité », Communication \& langages, n ${ }^{168}$, p. 43-56.

MATONTI Frédérique, 2017, Le genre présidentiel. Enquête sur l'ordre des sexes en politique, Paris, La Découverte.

MerCIER Arnaud, 2017, "Contestation et manipulations électorales en 140 signes », dans Le vote disruptif. Les élections présidentielle et législatives de 2017, P. Perrineau éd., Paris, Presses de Sciences Po.

NEVEU Érik, 1998, «La communication politique : un chantier fort de la recherche française », Polis, vol. V, nº 1, http://polis.sciencespobordeaux.fr/vol5n1/article3.html (consulté le 20 janvier 2018).

OGER Claire, 2003, "Communication et contrôle de la parole : de la clôture à la mise en scène de l'institution militaire», Quaderni, nº 52, p. 77-92.

OGer Claire, OlLivier-Yaniv Caroline, 2006, «Conjurer le désordre discursif. Les procédés de lissage dans la fabrication du discours institutionnel », Mots. Les langages du politique, no81, p. 63-77.

OLLIVIER-YANIV Caroline, 2003, «Des conditions de production politique : les écrivants des prises de paroles publiques ministérielles ", dans Argumentation et discours politiques. Antiquité grecque et latine, Révolution française, monde contemporain, S. Bonnafous et al. éd., Rennes, Presses universitaires de Rennes.

POUPARD Juliette, 2005, "Écrits d'écran : du mélange des genres», Communication \& langages, $\mathrm{n}^{0} 144$, p. 65-75.

RICHARD Arnaud, SANDRÉ Marion, 2013, "Le débat télévisé de primaires : un nouveau genre médiatique de discours politique en France», Nottingham French Studies, vol. LII, nº 2, p. 215-226. 
SANDRÉ Marion, 2017, «Discours primaire(s) », site de l'université de Toulon, http://www. univ-tln.fr/Marion-Sandre-discours-primaire-s.html (consulté le 20 janvier 2018).

- 2011, "Mimiques et politique. Analyse des rires et sourires dans le débat télévisé», Mots, Les langages du politique, $\mathrm{n}^{\circ}$ 96, p.13-28.

-2009, «Analyse d'un dysfonctionnement interactionnel - l'interruption - dans le débat de l'entre-deux-tours de l'élection présidentielle de 2007 ", Mots. Les langages du politique, no 89, p. 69-81.

THEVıot Anaïs, 2016, «Mettre en scène l'innovation politique en ligne. Analyse comparée de l'usage des réseaux sociaux au PS et à l'UMP pendant la campagne pour l'élection présidentielle de 2012 », séminaire Campagnes électorales et communication politique, organisé par P. Lefébure et F. Greffet à l'université Paris-Dauphine.

SAWICKI Frédéric, 2017, "L'épreuve du pouvoir est-elle vouée à être fatale au Parti socialiste? Retour sur le quinquennat de François Hollande», Pouvoirs. Revue française d'études constitutionnelles et politiques, $\mathrm{n}^{0} 163$, p. 27-41.

TREILLE Éric, 2013, «Primary colors. L'élection présidentielle à l'épreuve des primaires socialistes", dans Présidentielle 2012. Une communication politique bien singulière, P. J. Maarek éd., Paris, L'Harmattan.

TROGNon Alain, LARRUE Janine, 1994, «Les débats politiques télévisés », dans Pragmatique du discours politique, A. Trognon, J. Larrue éd., Paris, Armand Colin.

Vıon Robert, 2006, "Reprise et modes d'implication énonciative», La linguistique, vol. XLII, n², p. 11-28.

\section{Résumé / Abstract / Compendio}

\section{Une minute pour convaincre. L'expression politique à l'épreuve des débats télévisés des primaires de 2016-2017}

Le contrôle très strict de l'organisation des débats télévisés des primaires par LR et le PS en 2016 et 2017 - 1 minute par intervention et 15 minutes par candidat - résume l'ambiguïté de cette configuration médiatique et politique inédite qui doit à la fois départager et unir. L'énonciation de petites phrases s'est inscrite dans des stratégies politiques de rassemblement et d'évitement des faux pas, laissant majoritairement aux outsiders l'usage des formules polémiques.

Mots-clés : primaires, débats télévisés, campagnes électorales, communication politique, internet

\section{One minute to convince. Political expression and the test of televised debates of the 2016-2017 French primaries}

The very strictly controlled organization of the televised debates during the primaries of the French parties Les Républicains and the Parti socialiste in 2016 and 2017 -1 minute per intervention and 15 minutes per candidate-summarizes the ambiguity of this new media and political configuration which must both decide and reunite. The utterance of soundbites was part of political strategies of bringing people together and avoiding missteps, leaving mostly to outsiders the use of polemical formulas.

Keywords: primaries, televised debates, election campaigns, political communication, internet 
Un minuto para convencer. Expresión política a prueba de los debates televisados de las primarias 2016-2017

El control muy estricto de la organización de los debates televisados de las primarias por LR y el PS de 2016 y 2017 -1 minuto por intervención y 15 minutos por candidatoresume la ambigüedad de esta nueva configuración mediática y política que debe a la vez decidir y reunir. La formulación de frases cortas formaba parte de estrategias políticas de recopilación y evitación de errores, dejando a los outsiders el uso de fórmulas polémicas.

Palabras claves: elecciones primarias, debates televisados, campañas electorales, comunicación política, internet 